BULL. AUSTRAL. MATH. SOC.

VOL. $14(1976), 321-324$.

\title{
On a problem of Hahn
}

\section{W.A. Coppel}

It is shown that for any almost periodic linear differential system asymptotic stability and uniform stability together imply uniform asymptotic stability.

The question was asked by Hahn ( $[3]$, p. 202) whether, for almost periodic linear differential equations, asymptotic stability implies uniform stability. Conley and Miller [1] showed, by a fairly complicated example, that the answer was "no". The object of the present note is to point out that for an almost periodic linear differential equation asymptotic stability and uniform stability on the half-line $R^{+}$together imply uniform asymptotic stability on the whole line $R$. This follows without difficulty from other recent work, but the present strong and convenient formulation seems to have been overlooked. An immediate consequence is that if $a(t)$ is any almost periodic function with mean value 0 such that

$$
\int_{0}^{t} a(s) d s \rightarrow \infty \text { as } t \rightarrow \infty,
$$

then the scalar differential equation

$$
x^{\prime}=-a(t) x
$$

is asymptotically stable but not uniformly stable. For example (see [2], p. 72), one can take

$$
a(t)=\sum_{n=1}^{\infty} n^{-3 / 2} \sin \pi t / n
$$

Let

Received 14 January 1976. 


$$
x^{\prime}=A(t) x
$$

be any linear differential equation which is uniformly stable on the whole line $R$. Then there exists a constant $K>0$ such that, for every solution $x(t)$ of (1),

$$
|x(t)| \leq K|x(s)| \text { for }-\infty<s \leq t<\infty .
$$

It follows that if there exists a sequence $t_{n} \rightarrow-\infty$ such that $\left|x\left(t_{n}\right)\right| \rightarrow 0$ then $x(t) \equiv 0$. On the other hand, if there exists a sequence $t_{n} \rightarrow \infty$ such that $\left|x\left(t_{n}\right)\right| \rightarrow 0$ then $|x(t)| \rightarrow 0$ as $t \rightarrow \infty$. Hence either

$$
\inf |x(t)|>0
$$

or $|x(t)| \rightarrow 0$ as $t \rightarrow \infty$. Again, if

$$
\sup |x(t)|=\infty
$$

then, since $x(t)$ is bounded for $t \geq 0$, there exists a sequence $t_{n} \rightarrow-\infty$ such that $\left|x\left(t_{n}\right)\right| \rightarrow \infty$ and hence $|x(t)| \rightarrow \infty$ as $t \rightarrow-\infty$.

If, in addition, the coefficient matrix $A(t)$ is almost periodic then Nakajima [4] has shown that $x(t) \neq 0$ and

$$
\sup |x(t)|<\infty
$$

imply

$$
\inf |x(t)|>0 \text {. }
$$

Suppose now that $A(t)$ is almost periodic and (I) is uniformly stable on the half-line $R^{+}$. Then there exists a constant $K>0$ such that, if $X(t)$ is the fundamental matrix of (1) for which $X(0)=I$, then

$$
\left|X(t) X^{-1}(s)\right| \leq K \text { for } 0 \leq s \leq t<\infty \text {. }
$$

Let $B(t)$ be any almost periodic function in the hull of $A(t)$. Then there exists a sequence $t_{n} \rightarrow \infty$ such that $A\left(t+t_{n}\right) \rightarrow B(t)$ uniformiy on $R$. If $Y(t)$ is the fundamental matrix of the equation

$$
y^{\prime}=B(t) y
$$

for which $Y(0)=I$, then

$$
Y(t)=\lim _{n \rightarrow \infty} X\left(t+t_{n}\right) X^{-1}\left(t_{n}\right)
$$


for every real $t$ and hence

$$
\left|Y(t) Y^{-1}(s)\right| \leq K \text { for }-\infty<s \leq t<\infty .
$$

Thus (2), and in particular (1), is uniformly stable on the whole line $R$.

Assume (2) has a non-trivial bounded solution $y(t)$. Then, by Nakajima's result, inf $|y(t)|>0$. We have $B\left(t-t_{n}\right) \rightarrow A(t)$ uniformly on $R$, and by restricting attention to a subsequence we may suppose that $y\left(-t_{n}\right) \rightarrow \xi \neq 0$. Hence $y\left(t-t_{n}\right) \rightarrow x(t)$ for every real $t$, where $x(t)$ is the solution of (l) such that $x(0)=\xi$. Evidently $x(t)$ is bounded and $\inf |x(t)|>0$.

It follows that if the equation ( 1 ) is also asymptotically stable then no equation (2) has a nontrivial bounded solution. Therefore, by a fundamental result of Sacker and Sell [5], the equation (1) possesses an exponential dichotomy on $R$. Since $X(t)$ is bounded for $t \geq 0$ this is possible only if there exist positive constants $L, \alpha$ such that

$$
\left|X(t) X^{-1}(s)\right| \leq L e^{-\alpha(t-s)} \text { for }-\infty<s \leq t<\infty .
$$

In other words, the equation (I) is uniformly asymptotically stable on $R$.

\section{References}

[1] C.C. Conley and R.K. Miller, "Asymptotic stability without uniform stability: almost periodic coefficients", J. Differential Equations 1 (1965), 333-336.

[2] W.A. Coppel, Stability and asymptotic behavior of differential equations (Heath, Boston, 1965).

[3] Wolfgang Hahn, "The present state of Lyapunov's direct method", Nonlinear problems, 195-205 (Proceedings of a Symposium conducted by the Mathematics Research Center, United States Army, University of Wisconsin, Madison, 1962. University of Wisconsin Press, Madison, 1963).

[4] Fumio Nakajima, "Separation conditions and stability properties in almost periodic systems", Tôhoku Math. J. 26 (1974), 305-314. 
[5] Robert J. Sacker and George R. Sell, "Existence of dichotomies and invariant splittings for linear differential systems $I^{\prime \prime}, J$. Differential Equations 15 (1974), 429-458.

Department of Mathematics, Institute of Advanced Studies, Australian National University, Canberra, ACT. 